\title{
Sarcopenia definitions: Critical issues
}

\author{
Giovanni Iolascon, Antimo Moretti
}

In the last decades, there has been a progressive ageing of the population along with an increased prevalence of many age-related diseases that result in declined functional performance in older people thus leading to falls, fractures, disability, poor health-related quality of life (HRQoL) and increased mortality. After 25 years of age skeletal muscle mass undergoes a gradual decline [1], and until 80 years it is estimated a loss of $30 \%$ of muscle mass and $20 \%$ of cross sectional area (CSA) related to the reduction in the number and size of muscle fibers, particularly of type II fibers, probably because older people did not regularly perform vigorous physical activity [2]. Sarcopenia is one of the most relevant clinical and social health age-related condition, and identifies the muscle deconditioning characterized by loss of both muscle mass and function [3]. Since its appearance in the scientific literature, sarcopenia represented a topic of great interest for research. Initially, defined as sarcomalacia [4], referring purely to the structural component of the condition, several operational definitions and diagnostic cut-offs were subsequently proposed until the Centers for Disease Control and Prevention (CDC) recently assigned an ICD-10-CM code (M62.84) to the age-related sarcopenia [5]. However, the debate about both universal definition and accepted diagnostic criteria is still open, with major implications concerning endpoints identification in clinical trial for novel therapeutic approaches to muscle wasting diseases.

\section{Giovanni lolascon ${ }^{1}$, Antimo Moretti ${ }^{2}$}

Affiliations: ${ }^{1}$ Professor of Physical and Rehabilitation Medicine, Department of Medical and Surgical Specialties and Dentistry, University of Campania Luigi Vanvitelli, Naples, Italy; ${ }^{2}$ Physiatrist, Department of Medical and Surgical Specialties and Dentistry, University of Campania Luigi Vanvitelli, Naples, Italy.

Corresponding Author: Giovanni Iolascon, Via de Crecchio, 4, 80138 Naples, Italy; Email: giovanni.iolascon@gmail.com

Received: 19 December 2017

Published: 23 January 2018
The first consensus definition of sarcopenia was proposed in 2010 by the European Working Group on Sarcopenia in Older People (EWGSOP) [6] that also identified the assessment tools to investigate the key outcomes: muscle mass, strength and physical performance. Indeed, the EWGSOP recommended considering low muscle mass and muscle weakness or poor physical performance to diagnose sarcopenia. Moreover, both the EWGSOP and the International Working Group on Sarcopenia (IWGS) [7] focused also on the negative role of increased adipose tissue on the muscle quality with significant implications in terms of mobility limitation and increased disability in patients with sarcopenic obesity, characterized by the combination of obesity and muscle wasting. Furthermore, the same working groups pointed out that sarcopenia and cachexia are two different conditions, although they share several pathophysiological and clinical mechanisms, and cachexia may be a component of sarcopenia.

However, although the debate about how best to define this condition is still ongoing, a consensus on the diagnostic tools to be used for the assessment of the outcomes that characterize sarcopenia seems to be almost reached. To evaluate muscle mass, different imaging techniques analyzing body composition are available, including computed tomography (CT) scan, magnetic resonance imaging (MRI) scan, and dual-energy X-ray absorptiometry (DXA). The CT scan and MRI scan allow a precise analysis of different body tissues and are considered the gold standard for the measurement of muscle mass. However, high costs, poor availability and high exposure to ionizing radiation (for CT), are limiting factors to their use in clinical practice. The DXA total body scan is a particularly intriguing technique both for research and clinical practice, exposing the patient to a relative low dose of ionizing radiation and providing exhaustive information about body composition. Moreover, according to several sarcopenia research groups [EWGSOP, IWGS, and Foundation for the National Institutes of Health (FNIH) Biomarkers Consortium Sarcopenia Project] [8], DXA is a valid and reliable method to measure muscle mass. Another tool that can be useful for estimating the skeletal muscle mass 


\section{EDORiUM Journals}

in some setting, as in the case of bedridden patients, is the bioelectrical impedance analysis (BIA), a low-cost, easy to use and portable instrument. However, considering its limited validity, BIA could be used as screening method to assess muscle mass [9].

The first diagnostic criteria for defining low muscle mass in sarcopenic patients were those proposed by Baumgartner et al. [10] which introduced the skeletal muscle mass index (SMI) calculated as the ratio between appendicular skeletal muscle mass (ASM), measured by DXA, and the square of the height in meters (ASM/ $\mathrm{h}^{2}$ ). Skeletal muscle mass index values lower than 2 SD compared to those of a young population were considered for the diagnosis of sarcopenia. To date, the FNIH Sarcopenia Project recommends the use of the ratio between lean appendicular mass and body mass index (ALM/BMI), and proposed the use of evidence-based cut-points ( 0.789 for males and 0.512 for women) based on a large and diverse population, to define low muscle mass.

Although lower limbs play a key role in physical performance, the assessment of overall muscle strength in sarcopenic patients requires a handheld dynamometer, a transportable, low cost and easy to use tool, measuring the handgrip strength that is well correlated with physical performance [11]. Moreover, reduced handgrip strength is predictive of poor mobility, reduced muscle mass and disability [12]. In particular, a significant reduction of muscle strength is defined by a maximal handgrip strength (on 3 trials) $<26 \mathrm{~kg}$ in males and $<16 \mathrm{~kg}$ in females, as defined by the FNIH Sarcopenia Project [8].

Several methods assessing physical performance are available, including the Short Physical Performance Battery (SPPB) and the gait speed. The SPPB investigates balance, walking, strength and endurance by performing three tasks: to stand for 10 seconds with parallel feet, in semi-tandem position (toe placed sideways to heel) and tandem (toe posterior to the heel); the time taken to cover 4 or 6 meters (gait speed); the sit to stand test. A SPPB score $\leq 8$ is defined as poor muscle performance, according to the EWGSOP [6]. However, further working groups on sarcopenia $[7,8]$, suggest that the measurement of gait speed, already included in the SPPB, might be sufficient to identify an impairment of muscle performance both in clinical practice and research. In particular, walking speed slower than $0.8 \mathrm{~m} / \mathrm{s}$ should be considered as a sign of mobility limitation.

If on one hand the EWGSOP defines three different stages of sarcopenia (presarcopenia, sarcopenia, and severe sarcopenia) [6], which require at least reduced muscle mass with or without poor muscle function, there is a growing interest towards the concepts of sarcopenia with limited mobility [13] and skeletal muscle function deficit (SMFD) [14], which suggest, according to a much more practical, clinically meaningful, and ecological approach, to evaluate the physical performance as starting point and then move on to assessment of muscle strength and, finally, in case of impairment of muscle function, to the measurement of skeletal muscle mass. Indeed, the impairment of muscle function is not exclusively caused by the loss of muscle mass, but other pathophysiological factors are involved in age-related impairments of muscle function, such specific neurogenic factors, muscle quality, or systemic conditions (e.g., chronic inflammatory diseases or end-stage organ failure). Furthermore, evidence suggests that the relationship between muscle performance, strength and mass is not linear in older adults [15]. In particular low muscle mass alone is weakly associated with mobility limitation [16], while dynapenia, synonymous with muscle weakness, is strongly associated with poor muscle performance and disability [17].

In light of this perspective, a functional-oriented approach to the person with sarcopenia is recommended. According to the European Society for Clinical and Economic Aspects of Osteoporosis and Osteoarthritis (ESCEO), the International Classification of Functioning, Disability and Health (ICF) framework of the World Health Organization (WHO) based on the use of a common language for the definition and measurement of functioning and disability, in order to improve communication among different health-care providers, should be a useful tool to investigate the functioning of sarcopenic patients [18]. Indeed, the ESCEO proposed the ICF approach identifying a core set of domains within the core areas of the disease, considering the clinical manifestations (muscle mass, strength and performance) as ICF body functions and structures, falls, ADL disability, mobility, and HRQoL as ICF activity and participation, and resource use (costs) as environmental factors. Therefore, although primary endpoints might vary across different trials, the ICF conceptual model represents a viable strategy to identify both target population definitions and a core outcome set of potential endpoints to be addressed in research studies for new treatments of sarcopenia, thus enhancing the level of evidence.

Keywords: Aging, Sarcopenia, Skeletal muscle

\section{How to cite this article}

Iolascon G, Moretti A. Sarcopenia definitions: Critical issues. Edorium J Disabil Rehabil 2018;4:8-11.

Article ID: 100035Do5GI2018

$* * * * * * * * *$

doi: 10.5348/Do5-2018-36-ED-2 


\section{EDORiUM Journals}

Edorium J Disabil Rehabil 2018;4:8-11.

lolascon et al. 10

www.edoriumjournals.com/ej/dr

\section{REFERENCES}

1. Lexell J, Taylor CC, Sjöström M. What is the cause of the ageing atrophy? Total number, size and proportion of different fiber types studied in whole vastus lateralis muscle from 15 - to 83 -year-old men. J Neurol Sci 1988 Apr;84(2-3):275-94.

2. Rolland Y, Czerwinski S, Abellan Van Kan G, et al. Sarcopenia: Its assessment, etiology, pathogenesis, consequences and future perspectives. J Nutr Health Aging 2008 Aug-Sep;12(7):433-50.

3. Brioche T, Pagano AF, Py G, Chopard A. Muscle wasting and aging: Experimental models, fatty infiltrations, and prevention. Mol Aspects Med 2016 Aug;50:56-87.

4. Rosenberg I. Summary comments: Epidemiological and methodological problems in determining nutritional status of older persons. Am J Clin Nutr 1989;50:1231-3.

5. Falcon LJ, Harris-Love MO. Sarcopenia and the new ICD-10-CM Code: Screening, staging, and diagnosis considerations. Fed Pract 2017 Jul 9;34(7):24-32.

6. Cruz-Jentoft AJ, Baeyens JP, Bauer JM, et al. Sarcopenia: European consensus on definition and diagnosis: Report of the European working group on sarcopenia in older people. Age Ageing 2010 Jul;39(4):412-23.

7. Fielding RA, Vellas B, Evans WJ, et al. Sarcopenia: An undiagnosed condition in older adults. Current consensus definition: Prevalence, etiology, and consequences. International working group on sarcopenia. J Am Med Dir Assoc 2011 May;12(4):24956.

8. Studenski SA, Peters KW, Alley DE, et al. The FNIH sarcopenia project: Rationale, study description, conference recommendations, and final estimates. J Gerontol A Biol Sci Med Sci 2014 May;69(5):547-58.

9. Michel JP. Sarcopenia: There is a need for some steps forward. J Am Med Dir Assoc 2014 Jun;15(6):37980.

10. Baumgartner RN, Koehler KM, Gallagher D, et al. Epidemiology of sarcopenia among the elderly in New Mexico. Am J Epidemiol 1998 Apr 15;147(8):755-63.

11. Al Snih S, Markides KS, Ottenbacher KJ, Raji MA. Hand grip strength and incident ADL disability in elderly Mexican Americans over a seven-year period. Aging Clin Exp Res 2004 Dec;16(6):481-6.

12. Working group on functional outcome measures for clinical trials. Functional outcomes for clinical trials in frail older persons: Time to be moving. J Gerontol A Biol Sci Med Sci 2008 Feb;63(2):160-4.

13. Morley JE, Abbatecola AM, Argiles JM, et al. Sarcopenia with limited mobility: An international consensus. J Am Med Dir Assoc 2011 Jul;12(6):4039.
14. Correa-de-Araujo R, Hadley E. Skeletal muscle function deficit: A new terminology to embrace the evolving concepts of sarcopenia and age-related muscle dysfunction. J Gerontol A Biol Sci Med Sci 2014 May;69(5):591-4.

15. Goodpaster BH, Park SW, Harris TB, et al. The loss of skeletal muscle strength, mass, and quality in older adults: The health, aging and body composition study. J Gerontol A Biol Sci Med Sci 2006 Oct;61(10):105964.

16. Newman AB, Kupelian V, Visser M, et al. Strength, but not muscle mass, is associated with mortality in the health, aging and body composition study cohort. J Gerontol A Biol Sci Med Sci 2006 Jan;61(1):72-7.

17. Schaap LA, Koster A, Visser M. Adiposity, muscle mass, and muscle strength in relation to functional decline in older persons. Epidemiol Rev 2013;35:5165.

18. Reginster JY, Cooper C, Rizzoli R, et al. Recommendations for the conduct of clinical trials for drugs to treat or prevent sarcopenia. Aging Clin Exp Res. 2016 Feb;28(1):47-58.

$* * * * * * * * *$

\section{Author Contributions}

Giovanni Iolascon - Substantial contributions to conception and design, Acquisition of data, Analysis and interpretation of data, Drafting the article, Revising it critically for important intellectual content, Final approval of the version to be published

Antimo Moretti - Substantial contributions to conception and design, Acquisition of data, Analysis and interpretation of data, Drafting the article, Revising it critically for important intellectual content, Final approval of the version to be published

\section{Guarantor of Submission}

The corresponding author is the guarantor of submission.

\section{Source of Support}

None

\section{Conflict of Interest}

Authors declare no conflict of interest.

\section{Copyright}

(C) 2018 Giovanni Iolascon et al. This article is distributed under the terms of Creative Commons Attribution License which permits unrestricted use, distribution and reproduction in any medium provided the original author(s) and original publisher are properly credited. Please see the copyright policy on the journal website for more information. 
Access full text article on other devices

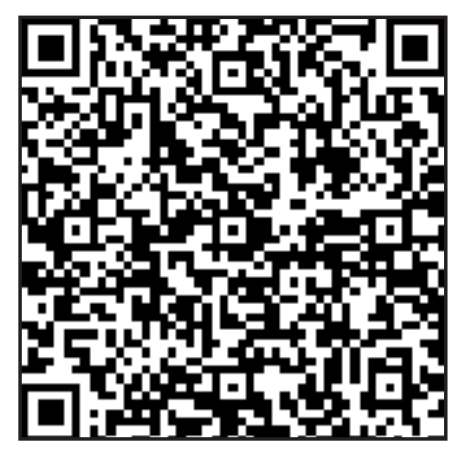

Access PDF of article on other devices

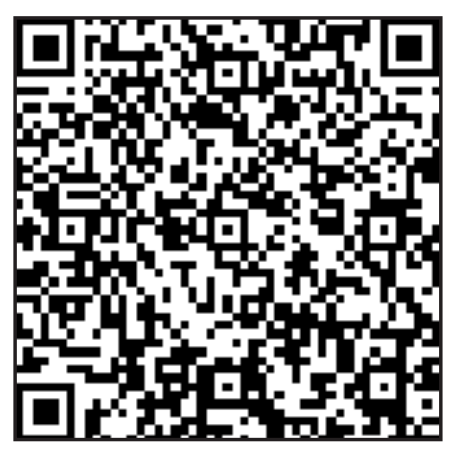

